\title{
Droit d'asile en france: état des lieux (partie 1)
}

\section{(Q) OpenEdition \\ Journals}

Édition électronique

URL : http://journals.openedition.org/conflits/369

DOI : $10.4000 /$ conflits.369

ISSN : $1777-5345$

Éditeur :

CCLS - Centre d'études sur les conflits lilberté et sécurité, L'Harmattan

Édition imprimée

Date de publication : 15 octobre 1996

ISSN : 1157-996X

\section{Référence électronique}

"Droit d'asile en france: état des lieux (partie 1) », Cultures \& Conflits [En ligne], 23 | automne 1996, mis en ligne le 15 mars 2006, consulté le 30 mars 2021. URL : http://journals.openedition.org/conflits/369 ; DOI : https://doi.org/10.4000/conflits.369

Ce document a été généré automatiquement le 30 mars 2021

Creative Commons License 


\section{Droit d'asile en france: état des lieux (partie 1)}

\section{DROIT D'ASILE EN FRANCE}

état des lieux ${ }^{1}$

Introduction

Le nouveau gouvernement a annoncé une refonte de la législation sur le statut des étrangers. En outre, le projet socialiste d'avril 1997 prévoyait de restaurer la spécificité $\mathrm{du}$ droit d'asile en sortant le chapitre relatif aux demandeurs d'asile de la législation relative à l'entrée et au séjour des étrangers. Pour comprendre ce qui devrait être modifié dans ce domaine, il est important de connaître avec précision les situations des personnes qui cherchent à obtenir une protection sur notre territoire. L'objet de ce texte est de décrire les procédures d'asile ${ }^{2}$ et de présenter les mesures qui entravent l'accès à celles-ci. Il est aussi important de montrer les conséquences sur la législation française des travaux de rapprochement des politiques d'asile au niveau européen.

Depuis plus d'une dizaine d'années, les questions relatives à l'asile sont de plus en plus systématiquement traitées par le biais du contrôle de l'immigration. La plupart des mesures visant à contrôler l'immigration, comme les visas, les sanctions à l'encontre des transporteurs et les accords de réadmission, constituent une menace pour des demandeurs d'asile, rarement en possession des documents requis pour l'entrée sur le territoire. En même temps, les États européens cherchent davantage à se protéger des personnes qui viennent demander asile sur notre continent qu'à se donner les moyens de les protéger. La recherche d'un pays tiers vers lequel renvoyer les demandeurs comporte le risque d'un renvoi in fine vers le pays d'origine, par des " renvois en chaîne " de pays tiers en pays tiers.

Au sein de l'Union européenne ou du groupe de Schengen, les États travaillent à la libre circulation des personnes sur le territoire commun. Ce projet implique des politiques communes en matière d'immigration, de contrôle aux frontières et de droit d'asile. L'application des mesures adoptées risque de remettre en cause l'exercice du droit d'asile. Certains pays d'Europe centrale sont déjà amenés à copier ces législations. En France, le gouvernement est allé jusqu'à réformer la Constitution pour subordonner l'exercice du droit d'asile aux accords de Schengen. Cette mesure exceptionnelle 
illustre les liens désormais indissociables entre les décisions arrêtées à l'échelon européen et leur transcription dans le droit interne.

En France, le nombre de demandeurs d'asile ne cesse de chuter et ils sont de moins en moins nombreux à obtenir la reconnaissance du statut de réfugié et la protection liée à la Convention de Genève. Les droits attachés à cette Convention sont importants : attribution d'une carte de résident de dix ans, droit au travail, au regroupement familial, etc. Pour certaines nationalités et dans certaines conditions, une protection précaire, l'asile territorial, est proposée en lieu et place de ce statut. De même, certains étrangers " déboutés " de leur demande d'asile sont parfois tolérés sur le territoire français lorsqu'un renvoi vers leur pays pourrait être dangereux mais ils sont laissés en situation irrégulière ${ }^{3}$.

Les associations européennes de défense des droits de l'Homme et du droit d'asile doivent continuer à agir pour promouvoir ensemble une harmonisation européenne respectueuse des engagements internationaux souscrits par les États et soucieuse d'offrir un refuge sûr à ceux qui fuient des menaces contre leur liberté ou leur sécurité physique. Ce texte souhaite y contribuer.

L'asile et la construction européenne

À la fin des années quatre-vingt, les États ont multiplié les forums de discussion dans le but de trouver de nouvelles formes de coopération, notamment dans le domaine de la coopération policière et la sécurité intérieure, de l'accès au territoire, de l'asile et de l'immigration. Les questions d'asile commençaient alors à être systématiquement mélangées à celles sur l'immigration. À ces réunions se retrouvaient des ministres ou des fonctionnaires des ministères concernés. Divers forums concernaient directement ou indirectement l'asile et l'immigration, comme les Groupes de Berlin ou de Vienne, les Consultations intergouvernementales ainsi que le Groupe de Trevi de lutte contre le terrorisme. C'est au niveau des groupes de concertation entre les membres de l'Union européenne ou du groupe de Schengen que les décisions les plus contraignantes ont été prises.

\section{La Convention d'application de l'Accord de Schengen}

Cadre général et rappel historique

L'Accord de Schengen (Luxembourg) " relatif à la suppression graduelle des contrôles aux frontières " a été signé le 14 juin 1985 par l'Allemagne, la Belgique, la France, le Luxembourg et les Pays-Bas. Le texte a été publié au Journal officiel le 5 août 1986. Les premiers États ont été rejoints plus tard par l'Italie (27 novembre 1990), l'Espagne et le Portugal (25 juin 1991), la Grèce (6 novembre 1992) puis l'Autriche, entrée dans l'Union européenne le 1er janvier 1995. Le 1er mai 1996, les cinq pays scandinaves étaient admis comme observateurs. Le Danemark, la Suède et la Finlande sont devenus membres le 19 décembre $1996^{4}$.

La Convention d'application de l'Accord de Schengen a été signée le 19 juin 1990 mais n'est entrée en vigueur que le 26 mars 1995 dans sept États membres: Allemagne, Belgique, Espagne, France, Luxembourg, Portugal et Pays-Bas 5 . Il s'agit d'un accord très large sur la coopération transfrontalière contenant des dispositions relatives à la création d'un espace de libre circulation des biens et des personnes ainsi que des mesures compensatoires à la suppression des contrôles aux frontières intérieures. Ces mesures passent par une coopération policière et judiciaire renforcée, notamment en matière d'accès au territoire commun (contrôle aux frontières extérieures, visa), de 
sécurité et de répression du trafic d'armes et de stupéfiants. Les dispositions relatives à l'asile ne représentent qu'une petite partie du texte (titre VII, articles 28 à 38). D'autres dispositions relatives aux visas, aux sanctions à l'encontre des transporteurs ou au Système d'Information Schengen (S.I.S.) ont également des répercussions sur la situation des demandeurs d'asile.

Les contrôles aux frontières intérieures ont été supprimés par tous les États membres, à l'exception de la France qui a demandé à ses partenaires une période probatoire de trois mois, au cours desquels certains contrôles étaient effectués de manière aléatoire aux frontières terrestres tandis qu'ils étaient levés aux aéroports. Après la vague d'attentats de l'été 1995, la France a décidé de rétablir les contrôles à toutes ses frontières, faisant appel à l'article 2.2 de la Convention qui prévoit cette possibilité " pour une période limitée " et " lorsque l'ordre public ou la sécurité nationale l'exigent. " Le 26 mars 1996, la France a levé les contrôles à l'exception des frontières avec la Belgique et le Luxembourg au motif que la législation sur les stupéfiants en vigueur aux Pays-Bas ne satisfait pas à ses exigences. Les pays partenaires de la France lui ont reproché de paralyser les mécanismes Schengen et de faire un usage abusif d'une clause dérogatoire dont les conditions d'application n'avaient jamais été clairement définies. En 1996, le groupe Schengen a finalement défini ses orientations relatives au recours à cet article 2.2.

Les liens avec l'organisation de l'Union européenne sont très marqués. Le préambule de la Convention de Schengen la replace d'emblée dans la perspective communautaire en faisant référence explicite au Traité de Rome et à l'Acte unique européen. En outre, seuls les États membres de l'Union européenne peuvent adhérer à Schengen. Il est par ailleurs clairement indiqué à l'article 134 de la Convention d'application que ses dispositions ne sont applicables " que dans la mesure où elles sont compatibles avec le droit communautaire ", tandis que les États Schengen se sont engagés à renoncer à appliquer les dispositions relatives à l'asile dès que la Convention de Dublin ${ }^{6}$ sera entrée en vigueur entre les États membres de l'Union européenne. Dans cette logique, les passerelles sont nombreuses entre le dispositif Schengen et le système prévu au sein de l'Union. Ces liens étroits s'expliquent par le fait que l'Accord de Schengen a été négocié par un petit groupe d'États membres de la Communauté européenne soucieux d'accélérer la création d'un espace commun de libre circulation et la mise en place des mesures compensatoires en attendant qu'aboutissent les travaux à quinze sur ces questions. À l'occasion de l'adoption du Traité d'Amsterdam les 16 et 17 juin 1997, le Sommet européen s'est accordé sur un protocole " incorporant l'acquis de Schengen dans le cadre de l'Union européenne ". Les textes adoptés par le groupe de Schengen ainsi que toutes les décisions et déclarations adoptées par son Comité exécutif s'appliqueront entre les États membres à l'exception de l'Irlande et du Royaume-Uni (voir Le Traité d'Amsterdam).

Structure interne de Schengen

Le Comité exécutif

Le Comité exécutif a été institué pour veiller à la bonne application de la Convention de Schengen dans le domaine de l'asile comme dans les autres. Il se compose d'un délégué de chacun des pays membres et des pays admis comme observateurs. La France y est représentée par son ministre délégué aux Affaires européennes. Les décisions du Comité ne sont soumises à aucun contrôle parlementaire à l'exception de deux États, 
les Pays-Bas et l'Italie, dont les parlements ont obtenu, au moment de la ratification, de pouvoir examiner les projets de décisions avant leur adoption définitive.

En l'absence de tout organe juridictionnel habilité à interpréter la Convention de Schengen et de toute instance d'arbitrage, c'est le Comité exécutif qui tente de dégager une doctrine en observant la règle du consensus et en recherchant des solutions aux difficultés rencontrées. Cette formule est fort peu satisfaisante, aux dires même du Comité, qui estime que les travaux menés par des groupes d'experts en son sein ne compensent que très imparfaitement l'absence d'une juridiction chargée d'interpréter les dispositions de la Convention. Ce problème avait été soulevé à de nombreuses reprises lors des débats qui avaient précédé la mise en œuvre de la Convention.

Le Système d'Information Schengen (S.I.S.)

Le S.I.S., clé de voûte de l'ensemble du dispositif Schengen (titre IV), est un fichier informatisé qui contient, entre autres ${ }^{7}$, les données relatives aux étrangers signalés aux fins de non-admission dans un des pays membres. Les données intégrées au S.I.S. sont soumises aux législations nationales sur la protection des données. Les pays qui ne présentaient pas les garanties nécessaires se sont dotés d'une législation adéquate. L'hétérogénéité des données inscrites au S.I.S., différentes selon le pays, et la consultation systématique du fichier par les États dès le dépôt d'une demande d'asile peuvent avoir de graves conséquences pour le demandeur ${ }^{8}$. Un étranger verra son nom inscrit dans le S.I.S. si sa demande d'asile est rejetée en Allemagne mais pas si elle l'est en France.

À elles seules, l'Allemagne et la France occupent la quasi-totalité de l'espace du S.I.S. : selon les statistiques du Groupe Schengen de mars 1996, sur 508000 données relatives aux étrangers signalés aux fins de non-admission (article 96), 416000 avaient été inscrites par l'Allemagne et 74000 par la France. Le Groupe Schengen étudie actuellement les possibilités d'étendre la capacité du système.

Dispositions relatives à une politique commune d'accès au territoire

Politique commune de visas

La réalisation d'un espace commun implique une politique commune d'accès au territoire. L'article 20 de l'Accord de Schengen prévoit que " les Parties s'efforceront de réaliser l'harmonisation de leurs politiques en matière de visa ". Désormais, un État partie délivre un " visa Schengen " matérialisé par une vignette uniforme qui permet aux étrangers ${ }^{9}$ de circuler librement dans tout l'espace Schengen, moyennant une déclaration d'entrée sur le territoire national des autres pays ${ }^{10}$. Ce qui peut paraître un avantage présente aussi de gros inconvénients : si un visa délivré par l'un des États est valable dans tous les autres, un refus de délivrance de visa par un pays Schengen peut également signifier a contrario le refus par l'ensemble des pays membres.

L'entrée en vigueur de la Convention de Schengen dans sept pays a créé de nouvelles règles en matière de circulation. Le visa uniforme est réglementé par les articles 9 à 17 de la Convention et par une instruction commune non publiée ${ }^{11}$. En vertu des critères de détermination de l'État responsable, un titulaire d'un visa Schengen qui désire demander l'asile voit sa demande examinée dans le pays qui lui a délivré le visa. Il existe donc en raison de ce principe une limitation territoriale de fait en matière de circulation à l'égard des demandeurs d'asile.

Les nationalités soumises à l'obligation du visa uniforme sont recensées en une liste commune aux États Schengen de 133 pays. Pratiquement tous les pays dont les 
demandeurs d'asile sont originaires y figurent. L'exigence de visa, qui constitue déjà en elle-même une barrière souvent difficilement franchissable, associée aux mécanismes Schengen, est d'une efficacité redoutable : selon le rapport annuel du Groupe Schengen couvrant la période du 26 mars 1995 au 25 mars 1996, " les données fournies permettent de conclure que l'on assiste à une diminution, prévue, du nombre de demandes de visa depuis la mise en application de la Convention. En moyenne, ce nombre diminue de $17 \%$ ".

Sanctions à l'encontre des transporteurs

L'article 26 de la Convention de Schengen fait obligation aux États d'instaurer des sanctions à l'encontre des transporteurs qui acheminent par voie aérienne, maritime ou terrestre des passagers dépourvus de documents requis pour l'entrée sur le territoire commun. Les passagers non européens risquent d'être victimes de discriminations lors du contrôle des passeports car ils peuvent être considérés a priori comme des suspects. Ceux qui n'ont pas été autorisés à embarquer ne disposent d'aucun recours légal ou effectif. Or ces personnes peuvent être des réfugiés qui soit n'ont pu se procurer de passeports ou de visas, soit, à cause des critères stricts de visa, ont été contraints de se procurer de faux documents de voyage.

Le personnel des compagnies de transport est devenu, dans la pratique, auxiliaire de police alors qu'il n'a pas la compétence requise : la confidentialité du contrôle n'est pas garantie, la maîtrise par l'agent de la langue du demandeur n'est pas assurée, aucune formation attentive n'est souvent envisagée ou alors il s'agira d'une formation destinée à détecter le passager susceptible d'entraîner une amende. Aussi, le " contrôleur " préfère refuser l'embarquement des passagers qui sont ou qu'il présume être en situation irrégulière de peur que la compagnie soit frappée d'une sanction. En outre, l'utilisation de sociétés de sécurité chargées par les compagnies de transport de ces contrôles semblent se généraliser. Cette " sous-traitance " à des sociétés privées uniquement destinées à cette tâche paraît totalement incompatible avec un examen individuel attentif des risques que pourrait entraîner un refus d'embarquer.

Les sanctions infligées aux transporteurs, associées à l'obligation d'être muni d'un visa d'entrée, ont sans doute contribué à une extension des réseaux impliqués dans des activités d'immigration clandestine. Plus les conditions de départ et d'entrée sont devenues difficiles, plus les prix demandés par les trafiquants ont grimpé. Pour fuir leur pays, des réfugiés sont obligés de renoncer à utiliser les moyens de transport réguliers et sont contraints de chercher à entrer clandestinement ${ }^{12}$. Les conditions de transport infligées aux demandeurs par les passeurs transfrontaliers et l'accueil réservé aux frontières à ceux qui sont démunis de documents requis ont engendré bien souvent des situations humaines dramatiques. Il n'est pas rare de rencontrer des demandeurs démunis de toute ressource à la suite de la vente de leurs biens.

La responsabilité des transporteurs et la protection des réfugiés

Le Haut Commissariat des Nations unies pour les réfugiés (H.C.R.) a jugé que " les États ne respectent pas leurs obligations internationales envers les réfugiés lorsque les mesures telle l'imposition de sanctions aux transporteurs empêchent les réfugiés d'accéder aux procédures d'asile ".

L'exigence de visa et l'instauration des sanctions à l'encontre des transporteurs assimilent dans un même contexte migratoire les réfugiés et les autres étrangers. En ne procédant pas à une distinction claire entre ces deux catégories, la politique des visas entrave l'accès de demandeurs d'asile à la procédure de détermination du statut de 
réfugié. L'obligation de visa et des critères très stricts de délivrance de ce document poussent à l'irrégularité des personnes fuyant la persécution. Ces mesures empêchent certains réfugiés de fuir le pays où ils sont menacés ou persécutés et violent indirectement l'article 33 de la Convention de Genève sur le statut des réfugiés (principe $\mathrm{du}$ non-refoulement) en obligeant les transporteurs à leur refuser l'embarquement.

Certains experts en matière de réfugiés ${ }^{13}$ estiment que les lois sur la responsabilité des transporteurs violent le paragraphe 2 de l'article 13 de la Déclaration universelle des droits de l'Homme de 1948, le paragraphe 2 du Pacte international relatif aux droits civils et politiques de 1966, ainsi que le paragraphe 2 du Protocole $n^{\circ} 4$ à la Convention européenne de sauvegarde des droits de l'Homme et des libertés fondamentales de 1950. Ces trois dispositions garantissent le droit de toute personne de quitter n'importe quel pays, y compris le sien. Cette interprétation peut être nuancée puisque la loi sur la responsabilité des transporteurs n'empêche pas directement une personne de quitter son pays mais plutôt d'accéder à un autre pays. En fait, le véritable objectif n'est pas d'empêcher une personne de quitter son pays mais plutôt de réduire les possibilités d'accès aux pays de l'espace Schengen, y compris les personnes qui craignent de subir des persécutions dans leur pays.

L'obligation de visa d'entrée ainsi que la loi infligeant des sanctions à l'encontre des transporteurs ont fait l'objet de critiques dans une recommandation $\left(\mathrm{n}^{\circ} 1163\right)$ de l'Assemblée parlementaire du Conseil de l'Europe de 1991 relative à l'arrivée de demandeurs d'asile dans les aéroports européens, qui estime que: " les conditions d'octroi du visa étant devenues plus strictes, les demandeurs d'asile ont de plus en plus souvent de faux documents de voyage, car il est difficile à un véritable demandeur d'asile fuyant la persécution d'obtenir ces documents légalement ". Elle souligne ensuite que les lois qui visent à infliger " [...] aux compagnies aériennes des sanctions [...] portent atteinte aux principes fondamentaux de protection des réfugiés et au droit des réfugiés de demander l'asile, tout en mettant les transporteurs lourdement à contribution sur les plans juridique, administratif et financier, et en déchargeant les services de l'immigration de leurs responsabilités ".

Dispositions relatives à l'asile

Un seul État responsable de l'examen d'une demande d'asile

La Convention de Schengen définit la demande d'asile comme une demande présentée par un étranger " en vue d'obtenir sa reconnaissance en qualité de réfugié conformément à la Convention de Genève ".

L'espace Schengen constituant un territoire commun entre les États membres, la Convention énonce le principe de la responsabilité d'un seul de ces États pour l'examen d'une demande d'asile, celui qui aura pris la plus grande part de responsabilité dans son entrée sur ce territoire. Néanmoins, l'article 29.2 prévoit que l'obligation de traiter une demande " n'entraîne pas celle d'autoriser dans tous les cas le demandeur à pénétrer ou à séjourner sur son territoire " et que " Toute partie contractante conserve le droit de refouler ou d'éloigner [...] un demandeur vers un pays tiers ". Cette disposition est inquiétante et ouvre la porte aux pires abus.

La Convention définit les critères permettant d'établir la responsabilité d'un État ${ }^{14}$. D'une manière générale, elle incombe à celui qui a délivré le visa ou le titre de séjour produit par le demandeur ${ }^{15}$. En l'absence de documents probants, le pays responsable est celui par les frontières extérieures duquel l'étranger a pénétré dans l'espace 
Schengen ou, en dernier ressort, celui auprès duquel la demande a été présentée. Deux listes permettent d'aider à établir le passage éventuel du demandeur par un État Schengen : la liste A recense les éléments objectifs apportant la preuve formelle ${ }^{16}$, la liste B des " éléments indicatifs " permettant de supposer ou de soupçonner ce passage ${ }^{17}$. Lorsqu'un État soupçonne ou établit qu'un autre État Schengen est responsable, il saisit ce second État pour lui demander de reprendre en charge le demandeur. Si la réponse est positive, le transfert - volontaire ou forcé - de l'intéressé aura lieu vers ce pays.

Des dispositions dérogatoires autorisent un État autre que celui dont la responsabilité est établie à traiter la demande d'asile " pour des raisons particulières tenant notamment au droit national " (article 29.4) ou " pour des raisons humanitaires fondées notamment sur des motifs familiaux ou culturels " (article 36). En avril 1997, le Comité exécutif a précisé les situations envisagées (cas de maladie grave, de handicap lourd, de personnes âgées à charge ou de mineurs séparés de leurs parents, grossesse ou naissance). Enfin, l'État qui a reconnu à un étranger le statut de réfugié et lui a accordé le droit au séjour examine la demande présentée par un membre de sa famille si les intéressés en sont d'accord ${ }^{18}$. Ces dispositions dérogatoires sont rarement appliquées.

Les conséquences pour les demandeurs d'asile

La conséquence essentielle pour les demandeurs d'asile est l'impossibilité de choisir leur pays d'accueil. Seul le critère objectif du pays qui a délivré le visa ou par lequel il pénètre dans l'espace commun est retenu. Les dispositions dérogatoires " à titre humanitaire " sont rarement appliquées et la mise en œuvre mécanique des dispositions conduit à des situations absurdes : des familles éparpillées, soit que leurs membres produisent des documents délivrés par différents pays Schengen, soit qu'ils ont suivi des itinéraires différents; des demandeurs renvoyés vers un pays dont ils ne parlent pas la langue ou avec lequel ils n'ont aucun lien familial, culturel ou affectif. Il est impératif que les États mettent fin à ces situations en faisant du respect de la vie de famille un critère contraignant dans la détermination du pays responsable.

L'absence de recours suspensif en cas de refus d'admission et le risque que la demande, en vertu des législations nationales, ne soit jamais examinée au fond sont vivement critiqués par les associations et le H.C.R. Certains États se contentent en effet d'une étude rapide de la recevabilité de la demande, sans examen au fond, sans même faire jouer les mécanismes Schengen ou sans donner à une personne réadmise à la demande d'un autre État Schengen l'accès à la procédure normale de détermination ${ }^{19}$. L'application, notamment par l'Allemagne et l'Espagne, des principes du " pays tiers sûr de premier accueil " et du " pays sûr d'origine " permet à ces États de déclarer une demande d'asile " manifestement infondée ". Le demandeur y sera renvoyé sans avoir eu droit à un examen sur le fond par un État Schengen. En outre, seules les législations néerlandaise et allemande, sous certaines conditions, accordent un droit de recours suspensif contre une décision de transfert vers un autre État Schengen.

<!--SPIP--> A l'usage, il s'avèreextrêmement difficile de reconstituer l'itinéraire d'un demandeur d'asile lorsqu'aucun document ne l'atteste. La France a tendance à soupçonner a priori le passage par un pays tiers et fait appel à la liste B (cf. ci-dessus) de manière beaucoup plus systématique que ses partenaires, qui s'en tiennent à des critères plus stricts et ne transmettent ou n'acceptent des demandes de reprise en charge que lorsque des éléments suffisamment probants étayent la requête. Tous les pays ont légiféré sur la mise en œuvre de Schengen mais selon des schémas très 
différents. La France a cadré de très près l'application du dispositif en instaurant une procédure spécifique où les instances de la procédure de détermination n'interviennent pas, tandis que d'autres pays, comme l'Allemagne et les Pays-Bas, ont conservé la procédure normale, en créant une structure spéciale chargée du traitement des dossiers Schengen au sein de l'instance de détermination. L'Espagne a mis en place une procédure proche du système français, alors que le Portugal, en l'absence de réglementation spécifique, applique une procédure complexe et mal comprise des différentes administrations.

L'harmonisation au niveau de la Communauté européenne

Le fonctionnement du Groupe ad hoc immigration

La signature, en février 1986, de l'Acte unique européen par les pays membres de la Communauté économique européenne a confirmé la volonté des États de créer un espace de libre circulation des biens et des personnes en Europe, ce qui impliquait la suppression des contrôles aux frontières intérieures de la Communauté. Les États ont immédiatement pris conscience des conséquences de cette décision sur l'entrée et le séjour des ressortissants non communautaires et de la nécessité de rapprocher leur politique d'immigration.

Le Groupe ad hoc immigration (G.A.H.I.), créé en 1986, fut un forum de coopération intergouvernementale agissant en dehors du système communautaire. Il était composé des ministres des États membres (douze à l'époque) compétents en matière d'asile et d'immigration, les ministres de l'Intérieur ou de la Justice. Dans le contexte communautaire, le terme " intergouvernemental " désigne des structures de concertation qui ne sont pas intégrées aux mécanismes communautaires mais qui regroupent des représentants des États membres.

Dans la pratique, le G.A.H.I. a œuvré dans le plus grand secret, ses travaux n'étant soumis à aucun des mécanismes de contrôle parlementaire ou judiciaire prévus par le Traité de Rome. Pourtant, ses décisions et résolutions, bien qu'issues d'un processus de concertation sans aucune transparence ni contrôle démocratique, gagnaient une certaine légitimité communautaire par leur adoption formelle par le Conseil européen, instance politique suprême de l'Union. En outre, les États étaient vivement encouragés à mettre rapidement en œuvre les mesures préconisées, les textes contenant généralement une échéance d'application. S'ils ne sont pas contraignants juridiquement, les textes adoptés par le G.A.H.I. le sont donc politiquement. Ce double système, entaché d'un lourd déficit démocratique, a longtemps été dénoncé par les associations mais aussi par les institutions européennes qui y voyaient un mépris affiché de leur rôle institutionnel et une perversion de l'esprit du Traité de Rome. Les mécanismes mis en place par le Traité de Maastricht n'ont que très imparfaitement comblé ce déficit.

Le traité de Maastricht et l'Union européenne

Cinq ans après l'Acte unique européen, le Traité sur l'Union européenne (T.U.E.), dit aussi Traité de Maastricht, adopté par les chefs d'États et de gouvernement au sommet européen de Maastricht en décembre 1991 et entré en vigueur le 1er novembre 1993, prévoit la suppression complète des contrôles aux frontières intérieures et la libre circulation des biens et des personnes dans l'espace communautaire. Ces mesures s'accompagnent d'une coopération policière, judiciaire et douanière renforcée et impliquent, à terme, des politiques communes en matière d'accès au territoire, d'immigration, de traitement des demandes d'asile et de contrôle aux frontières 
extérieures. Il y a donc continuité absolue entre les travaux du Groupe ad hoc immigration et l'action menée dans le cadre du T.U.E.

Le Traité de Maastricht regroupe trois grands domaines de coopération, couramment désignés sous le terme de " piliers ". Le premier pilier définit le rôle des institutions communautaires dont il reprend les compétences traditionnelles (union économique et monétaire, cohésion économique et sociale, citoyenneté de l'Union) mais qu'il élargit à la politique des visas, innovation importante qui entrouvre une petite brèche dans la souveraineté nationale des États en matière d'accès au territoire. Ce pilier central, désigné comme " Traité instituant la Communauté européenne ", est flanqué du deuxième pilier intitulé " Politique étrangère et de sécurité commune " (P.E.S.C.) et du troisième pilier couvrant les domaines " Justice et affaires intérieures " (J.A.I.). C'est dans le cadre du troisième pilier que sont traitées les questions liées à l'asile et à l'immigration, ainsi que la coopération policière, judiciaire et douanière.

Ces piliers constituent à eux trois l'Union européenne, mais seul le premier relève du droit communautaire. Les piliers II et III ne sont pas intégrés au système communautaire et les actions et décisions prises dans leur cadre sont donc toujours les fruits de la coopération intergouvernementale. Cependant, toutes les questions d'harmonisation européenne sont désormais regroupées dans un seul cadre juridique. C'est le mérite de Maastricht que d'avoir conféré à la coopération intergouvernementale un caractère plus institutionnel et plus unitaire. Tous les forums de coopération créés dans les années quatre-vingt pour traiter des questions ne relevant pas du droit communautaire ont été remplacés au sein du troisième pilier par trois Comités directeurs, le Comité I traitant des questions d'asile et d'immigration. Ces comités sont réunis au sein d'un Comité de coordination prévu par l'article K.4 du Traité. Les diplomates du Comité des représentants permanents (COREPER) interviennent également dans les discussions pour assister le Conseil. De manière générale, le troisième pilier définit les politiques d'asile et d'immigration comme des " questions d'intérêt commun " et renforce les rôles de la Commission et du Parlement européens. Avec le Traité sur l'Union, les travaux d'harmonisation ont gagné en transparence, même si l'on est encore très loin d'un véritable processus de consultation et de contrôle démocratique.

Le troisième pilier comprend également deux organes permanents: le Centre d'information, de réflexion et d'échanges en matière d'asile (C.I.R.E.A.) et le Centre d'information, de réflexion et d'échanges en matière de franchissement des frontières et d'immigration (C.I.R.E.F.I.) en cours d'élaboration. Le C.I.R.E.A. est un lieu d'information et de concertation entre les Quinze. Il a pour objectif, notamment, de parvenir à une évaluation commune de la situation dans les pays d'origine des demandeurs d'asile. Il communique aux États européens des rapports confidentiels, produits principalement à partir d'informations d'ambassades, mais aussi à la suite de missions conjointes ou effectuées par un seul État membre dans les pays d'origine des demandeurs d'asile ou dans les pays tiers d'accueil20. Ces rapports sont ensuite utilisés par les organismes chargés de la détermination de la qualité de réfugié. Le C.I.R.E.A. compile et compare également les données concernant d'une part la reconnaissance au statut des demandeurs de ces pays, d'autre part les accords prévus avec les pays d'origine pour leurs renvois. Seuls les représentants de l'État ont accès aux données stockées par le C.I.R.E.A. Le secret qui entoure ses activités et son fonctionnement n'est pas fait pour rassurer les associations et les avocats, qui y voient la volonté des États 
d'utiliser ces informations confidentielles, et par conséquent invérifiables et non contestables, pour rapprocher leur politique d'asile.

Cette nouvelle construction européenne issue de Maastricht est peut-être un premier pas vers une architecture plus ouverte, où les questions relatives à l'asile et à l'immigration trouveraient leur place dans le système communautaire avec tous les garde-fous juridictionnels et parlementaires qu'il comporte. Leur " communautarisation ", rendue techniquement et juridiquement possible par l'effet combiné des articles $100 \mathrm{c}$ et $\mathrm{K} 9$ du Traité, dépend désormais de la seule volonté politique des États qui, pour certains, s'y opposent encore farouchement. Elle est encouragée depuis plusieurs années par bon nombre d'organisations non gouvernementales, ainsi que par la Commission et le Parlement européens, qui y voient le seul moyen de faire fonctionner efficacement les mécanismes du troisième pilier et de garantir un minimum de contrôle démocratique. Il ne s'agit pas de parer les institutions communautaires de toutes les vertus en matière de droits de l'homme, non plus que de penser que la " communautarisation " des questions relatives à l'asile garantira à coup sûr un accueil plus généreux et une meilleure protection des réfugiés en Europe. Tout au plus, peut-on espérer que la Commission et le Parlement européens pourront enfin jouer leur rôle et que cette solution est préférable à de mystérieuses négociations à huis clos. En revanche, certains observateurs craignent que cette communautarisation n'ait finalement pour effet de rendre contraignants des textes restrictifs, notamment ceux déjà adoptés par les instances intergouvernementales.

Les résultats des travaux d'harmonisation européenne

Le processus d'harmonisation européenne s'est déroulé en deux phases bien distinctes : jusqu'en 1993, les États, conscients de la diversité des procédures et des systèmes juridiques européens, ont d'abord tenté de rapprocher leurs politiques et leurs pratiques en matière d'accès au territoire. Ce choix reposait aussi sur la conviction qu'il était prioritaire de prendre des mesures compensatoires à la liberté de circulation. L'Europe communautaire, se voulant un espace sans frontières intérieures, est assimilable à un territoire commun, ce qui implique des règles communes d'accès à ce territoire et à la procédure de détermination de la qualité de réfugié.

C'est dans cet esprit qu'ont été adoptés, en décembre 1992, par les ministres siégeant au G.A.H.I. réunis à Londres puis par le Conseil européen d'Édimbourg, trois textes relatifs au caractère " manifestement infondé " d'une demande, au " pays tiers d'accueil " et au " pays sûr d'origine ". Les définitions communes proposées par ces textes ont été depuis intégrées à la législation de la plupart des États membres. Leur application permet d'instaurer une procédure de recevabilité de la demande d'asile ${ }^{20}$, et de procéder à des rejets à l'issue d'une procédure particulière, en général " accélérée ", et selon des critères ne relevant ni du fond de la demande ni par conséquent de la Convention de Genève. Ainsi, un passage ou un simple transit par un pays tiers extérieur à l'espace communautaire, un pays d'origine " où il n'existe généralement pas de risque de persécution " (dit aussi " pays sûr d'origine "), des déclarations considérées comme frauduleuses ou un récit " peu crédible " suffisent-ils à décider, parfois en quelques heures, qu'une demande est " manifestement infondée " ou à refuser l'entrée sur le territoire de l'État lorsque l'examen de recevabilité se fait à la frontière. L'économie générale des trois textes adoptés à Édimbourg, comme celle de l'ensemble des travaux d'harmonisation européenne, notamment en matière de visa, 
est de limiter autant que faire se peut l'admission des demandeurs d'asile sur le territoire commun.

Cette première phase achevée, les États européens travaillent, depuis 1994, à l'harmonisation des procédures et des critères de détermination de la qualité de réfugié. En mars 1995, les Quinze ont adopté une résolution sur " les garanties minimales que doivent présenter les procédures en matière d'asile ". En mars 1996, ils s'entendaient sur une position commune relative à " l'application harmonisée de la définition du terme réfugié au sens de l'article 1er de la Convention de Genève ". Ces derniers textes sont révélateurs de l'esprit qui préside actuellement aux travaux d'harmonisation, qui consiste bien plus à préserver les pratiques nationales qu'à adopter des normes communes de protection. L'échec, en 1996, des négociations sur le projet d'une position commune (devenu dans une version ultérieure " recommandation ") sur les conditions minimales de l'accueil des demandeurs d'asile illustre les difficultés des États à s'entendre sur des textes susceptibles d'élever le niveau de protection des demandeurs d'asile et des réfugiés.

Le Traité d'Amsterdam

Après les travaux de la Conférence intergouvernementale (C.I.G.) chargée de préparer la révision du Traité de Maastricht, le Sommet européen des 16 et 17 juin 1997 a adopté le Traité d'Amsterdam. Diverses décisions ont été prises dans le domaine de l'asile dont les conséquences seront à analyser attentivement. Un nouveau titre a été créé insérant dans le premier pilier les questions relatives à l'asile et à l'immigration. Le Conseil prévoit d'" arrêter dans les cinq ans qui suivent l'entrée en vigueur du présent Traité des mesures relatives à l'asile "21 22. De même, un protocole a été adopté " incorporant l'acquis de Schengen dans le cadre de l'Union européenne "; les textes adoptés par le groupe de Schengen, y compris toutes les décisions et déclarations adoptées par son Comité exécutif, s'appliqueront entre treize États ${ }^{23}$.

Un des enjeux de ce Sommet fut la discussion sur une proposition de l'Espagne visant à exclure ou à limiter le droit d'un ressortissant d'un pays de l'Union européenne de demander asile à un autre pays de l'Union. Ces demandes sont certes très peu nombreuses mais cette limitation est inquiétante car elle porte atteinte au respect de la Convention de Genève en introduisant une réserve géographique et la notion de pays sûr d'origine. Elle modifie par ailleurs, au niveau régional, une convention internationale, ce qui enfreint le droit international général. En outre, personne ne peut prévoir ce qui peut arriver dans les États de l'Union européenne d'aujourd'hui ou a fortiori demain dans une Union élargie. Enfin, cette disposition pourrait créer un dangereux précédent, d'autres États dans d'autres régions du monde pouvant s'en inspirer. Le H.C.R. et les associations ne s'y sont pas trompés, ils ont lutté contre ce projet avant le Sommet puis ont dénoncé son adoption.

Le Sommet a en effet adopté un protocole qui précise que " toute demande d'asile présentée par un ressortissant d'un État membre ne peut être prise en considération [...] par un autre État membre que dans les cas suivants ", notamment " si l'État membre prend des mesures dérogeant à ses obligations au titre de la Convention de sauvegarde des droits de l'homme " ou " si le Conseil a constaté [...] l'existence d'une violation grave et persistante par cet État ". Une déclaration est jointe: " Le présent protocole ne préjuge pas le droit de chaque État membre de prendre les mesures d'organisation qu'il juge nécessaires pour remplir ses obligations au titre de la convention de Genève ". Le protocole et la déclaration semblent contradictoires. La 
Belgique, quant à elle, a ajouté une déclaration selon laquelle " elle effectuera un examen individuel de toute demande d'asile présentée par un ressortissant d'un autre État membre ".

Conséquences en France des travaux d'harmonisation

Les conditions d'entrée et de séjour des étrangers en France sont régies par l'ordonnance du 2 novembre 1945. Ce texte a fait l'objet de plus de quarante modifications depuis 1945 en fonction des évolutions économiques et politiques. La dernière révision en date, la loi dite " Debré " du 24 avril 1997, a été adoptée à l'issue des nombreux rebondissements qui ont suivi les travaux menés au sein de l'Assemblée nationale par la Commission d'enquête sur l'immigration clandestine et le séjour irrégulier d'étrangers en France et la publication de ses recommandations en avril 1996. Finalement, la loi du 24 avril 1997 ne contiendra aucune disposition importante concernant le droit d'asile.

Une loi sur les sanctions

L'article 26 de la Convention de Schengen recommande aux États membres d'instaurer des sanctions à l'encontre des transporteurs qui achemineraient un passager démuni des documents de voyage ou du visa requis. Cette mesure a été intégrée à l'article 20 bis de l'ordonnance du 2 novembre 1945 par la loi du 26 février 1992 qui institue une amende administrative d'un montant maximum de 10000 francs.

La révision de la Constitution française

L'exemple des dispositions de la loi sur la maîtrise de l'immigration du 24 août et du 30 décembre 1993 et la révision de la Constitution illustrent les liens entre les décisions arrêtées à l'échelon européen et leur transcription dans le droit interne français.

Juillet 1993 : le Parlement a adopté la loi relative aux conditions d'entrée, d'accueil et de séjour des étrangers en France dite " loi Pasqua ", qui vient modifier l'ordonnance du 2 novembre 1945 relative aux conditions d'entrée et de séjour des étrangers en France. Un chapitre est consacré à l'asile, au sein duquel l'article 31 bis donne le pouvoir aux préfectures de refuser, dans certains cas, l'admission au séjour. L'article 31 bis 1 est la transcription en droit interne de la mise en application de la Convention de Schengen ${ }^{24}$.

13 août 1993 : décision du Conseil constitutionnel ; huit articles sont censurés. Pour censurer l'article 31 bis 1 , le Conseil s'est référé à l'alinéa 4 du préambule de la Constitution, selon lequel " Tout homme persécuté en raison de son action en faveur de la liberté a droit d'asile sur les territoires de la République ". Tout demandeur d'asile qui invoque cet alinéa 4 " doit être autorisé à demeurer provisoirement sur le territoire jusqu'à ce qu'il ait été statué sur sa demande " et doit pouvoir exercer les droits de la défense, ce qui implique la suspensivité du recours. Cette décision remettait partiellement en cause la Convention de Schengen (pourtant entérinée au moment de sa ratification en 1991), en transformant une faculté (article 29.4) en une obligation. La décision du Conseil n'étant pas compatible avec les engagements européens de la France, le gouvernement a décidé de réviser la Constitution.

25 novembre 1993: la loi constitutionnelle " relative aux accords internationaux en matière de droit d'asile " a introduit dans la Constitution un nouvel article prévoyant une exception à la stricte application du principe relatif à l'asile. Cette exception découle de l'application d'accords conclus entre États européens " déterminant leurs compétences respectives pour l'examen des demandes d'asile qui leur sont présentées 
". Le texte du nouvel article 53.1 de la Constitution stipule, néanmoins, que la France a toujours la possibilité - et non plus l'obligation - d'examiner et d'accepter une demande d'asile même si cette responsabilité ne lui incombe pas en application des traités européens - ce que la Convention de Schengen prévoit également - et même si la demande a déjà été examinée par un autre État.

30 décembre 1993: la loi du 30 décembre réintègre certaines dispositions, parfois réaménagées et, notamment, la première rédaction de l'article 31 bis 1 adoptée en juillet 1993: non-admission au séjour et impossibilité de saisir les instances de détermination de la qualité de réfugié si les dispositions des conventions de Schengen et de Dublin sont applicables. La loi modifie également la loi du 25 juillet 1952 portant création de ces instances et déclare ces organismes incompétents pour connaître ces demandes.

\section{NOTES}

1. Rédigé par Amnesty International Section Française et France Terre d'Asile pour la Commission de sauvegarde du droit d'Asile. Ce texte a été préparé grâce au travail de nombreux membres de nos associations, bénévoles, objecteurs de conscience, stagiaires, salariés, responsables. Des avocats spécialisés et d'autres associations membres y ont contribué par leurs commentaires. La rédaction et la réalisation ont été coordonnées par Patrick Delouvin et Pedro Vianna.

2. Il s'agit de la procédure à la frontière, la procédure visant à obtenir le statut de réfugié en application de la Convention de Genève et la procédure visant à obtenir l'asile territorial.

3. La circulaire du ministère de l'Intérieur du 24 juin 1997 prévoit de résoudre certaines de ces situations.

4. Un accord particulier, dit Accord de coopération, a été conclu le 19 décembre 1996 avec l'Islande et la Norvège, pays extérieurs à l'Union européenne, qui, juridiquement, ne peuvent pas être signataires de la Convention d'application mais qui bénéficieront de facto de ses dispositions par le biais de l'accord de libre circulation de l'Union nordique des passeports.

5. L'Italie et la Grèce ne présentaient pas encore les garanties minimales exigées en matière de protection des données et des contrôles aux frontières extérieures. Lors de la réunion du Comité exécutif du 19 décembre 1996, la volonté a été affirmée de créer les conditions préalables pour la mise en application de la Convention en Italie, en Grèce et en Autriche simultanément à partir du 26 octobre 1997.

6. La Convention " relative à la détermination de l'État responsable d'une demande d'asile présentée dans l'un des États membres des Communautés européennes ", signée à Dublin le 15 juin 1990, devrait entrer en application le 1er septembre 1997.

7. Le S.I.S. contient des données relatives à des personnes mais aussi à des objets volés, détournés ou égarés comme des véhicules, des armes à feu, des documents vierges ou des documents d'identité, ainsi que des billets de banque. 
8. En France, l'inscription au S.I.S. entraîne le refus de la délivrance de l'autorisation provisoire de séjour ou, dans certains cas, le retrait des documents de séjour et la mise sous " convocation Schengen ". Dans certains cas, des arrêtés de reconduite à la frontière ont été pris par des préfectures à l'encontre de certaines personnes signalées comme déboutées dans un autre pays Schengen, ce qui a été sanctionné à plusieurs reprises par les tribunaux administratifs.

9. Selon l'article premier de la Convention de Schengen, par étranger il faut entendre " toute personne autre que les ressortissants des États membres de la Communauté économique européenne ". Les ressortissants d'un autre État partie et ceux des autres États membres de la C.E.E. ne sont pas désignés comme des étrangers. Nuance qui explique la vocation de la Convention à s'étendre à l'ensemble des États de l'Union européenne.

10. Les modalités de la procédure de déclaration d'entrée sur le territoire ont fait l'objet en France d'une circulaire datée du 15 mars 1995.

11. Cette instruction commune a été adoptée par le Comité exécutif Schengen le 18 octobre 1993. Le Comité a ensuite adopté diverses dispositions complémentaires concernant notamment la prolongation et l'annulation.

12. Les méthodes sont diverses : achat de faux papiers, corruption de fonctionnaires, paiement de passeurs aux frontières, utilisation des mêmes faux documents d'identité successivement pour plusieurs demandeurs d'asile.

13. Conseil européen sur les réfugiés et les exilés (CERE) : A European Refugee Policy in the Light of Established Principles, Londres, 1994.

14. Ce principe, consacré par Schengen, a été repris au niveau de l'Union européenne dans la Convention de Dublin qui doit entrer en vigueur le 1er septembre 1997.

15. Si le demandeur est détenteur de plusieurs visas délivrés par des pays différents, l'État responsable est celui qui a délivré le visa ayant l'échéance la plus lointaine.

16. Existence d'un visa, d'un cachet d'entrée par des services d'immigration ou de documents de séjour.

17. Il s'agit de tickets de transports, notes de rendez-vous médicaux, notes d'hôtel, déclarations de l'intéressé, témoignages de passagers, etc.

18. Par "membre de la famille ", la Convention entend le conjoint, l'enfant de moins de dix-huit ans et, s'il s'agit d'un mineur de moins de dix-huit ans célibataire, du père et de la mère.

19. Cela est possible au moyen de l'application combinée des articles 29.2 et 29.4 de la Convention d'application.

20. Rapports rédigés sur les pays d'origine : Albanie, Angola, Arménie, Bulgarie, Chine, Éthiopie, Ghana, Guinée Conakry, Iran, Nigeria, Nord-Est et Nord-Ouest de la Somalie, Sri Lanka, Turquie, Zaïre. Rapport sur les réfugiés afghans au Pakistan.

21. Cela veut dire que, si elle est déclarée irrecevable, la demande ne sera pas examinée au fond.

22. Parmi ces mesures figurent les suivantes : "critères et mécanismes de détermination de l'État membre responsable de l'examen d'une demande, [...] normes minimales régissant l'accueil des demandeurs, [...] normes minimales concernant les conditions que doivent remplir les ressortissants de pays tiers pour pouvoir prétendre au statut de réfugié, [...] normes minimales concernant la procédure d'octroi ou de retrait du statut de réfugié, [...] normes minimales relatives à l'octroi d'une protection temporaire". 
23. Les quinze États membres de l'Union européenne à l'exception de l'Irlande et du Royaume-Uni.

24. Pour les détails sur l'article 31 bis, voir le chapitre Le traitement des demandes d'asile dans les préfectures, une procédure prioritaire.

INDEX

Mots-clés : asile, droit 